\title{
Cognitive efficiency declines over time in adults with Type 1 diabetes: effects of micro- and macrovascular complications
}

\author{
C. M. Ryan ${ }^{1}$, M. O. Geckle ${ }^{1}$, T. J. Orchard ${ }^{2}$ \\ ${ }^{1}$ Western Psychiatric Institute and Clinic, University of Pittsburgh School of Medicine, Pittsburgh, USA \\ ${ }^{2}$ Department of Epidemiology, University of Pittsburgh Graduate School of Public Health, USA
}

\begin{abstract}
Aims/hypothesis. Mild cognitive dysfunction is not uncommon in adults with Type 1 diabetes, but its pathogenesis remains unclear. Previous cross-sectional studies had suggested that microangiopathy might affect brain integrity and lead to 'central neuropathy.' To assess the relationship between changes in cognitive performance and the incidence of new micro- and macrovascular complications, 103 young and middle-aged adults (mean age: 40 yrs) with childhood-onset Type 1 diabetes were followed over a 7-year period, and were compared to 57 demographically-similar adults without diabetes. Methods. All subjects completed a comprehensive battery of neurocognitive tests on two occasions. Diabetic subjects also received repeated medical assessments to diagnose the onset of clinically significant complications.

Results. Relative to control subjects, diabetic adults showed significant declines on measures of psychomotor efficiency; no between-group differences were
\end{abstract}

evident on learning, memory, or problem-solving tasks. The development of proliferative retinopathy and autonomic neuropathy during the follow-up period predicted decline in psychomotor speed $(p<0.01)$, as did incident macrovascular complications $(p<0.05)$, systolic blood pressure at follow-up $(p<0.01)$, and duration of diabetes $(p<0.01)$.

Conclusion/interpretation. This study shows that cognitive efficiency may decline over time in diabetic adults, and that this neurocognitive change may be linked, at least in part, to the occurrence of complications like proliferative retinopathy and elevated blood pressure. Therapeutic interventions that reduce the risk of vascular complications may have a similarly beneficial effect on the brain and reduce the risk of neurocognitive dysfunction in diabetic patients. [Diabetologia (2003) 46:940-948]

Keywords Adults, type 1 diabetes, cognitive dysfunction, microvascular complications, macrovascular complications.
Received: 20 January 2003 / Revised: 24 March 2003

Published online: 18 June 2003

C) Springer-Verlag 2003

Corresponding author: Dr. C. M. Ryan, Western Psychiatric Institute and Clinic, University of Pittsburgh School of Medicine, 3811 O'Hara Street, Pittsburgh, PA 15213, USA

E-mail: ryancm@msx.upmc.edu

Abbreviations: AN, Autonomic neuropathy; BAEP, brainstem auditory evoked potential; CNS, central nervous system; CAD, coronary artery disease; DSST, digit symbol substitution test; DSP, distal symmetrical polyneuropathy; EDC, epidemiology of diabetes complications; MRI, magnetic resonance imaging; $\mathrm{ON}$, overt nephropathy; PVD, peripheral vascular disease; PDR, proliferative diabetic retinopathy; $\mathrm{rCBF}$, regional cerebral blood flow; WAIS-R, wechsler adult intelligence scale, revised.
Diabetes mellitus is associated with a greatly increased risk of mild brain dysfunction [1]. Adults with Type 1 diabetes may manifest impairments on neurocognitive tests requiring rapid decision-making and adaptive problem-solving skills [2], learning and memory [3], visuospatial analysis [4], and psychomotor efficiency [5]. Compared to their non-diabetic peers, they may also have increased rates of cortical atrophy [6] and more subcortical and brainstem lesions [7] on magnetic resonance imaging (MRI) scans, slower neural transmission times on brainstem auditory evoked potential (BAEP) studies [7] and more regional cerebral blood flow (rCBF) perfusion abnormalities [8]. 
The aetiology of this brain dysfunction remains controversial. Some writers have attributed these abnormalities to recurrent episodes of moderately severe hypoglycaemia [9, 10], but large-scale clinical trials have not found linkages between neurocognitive impairment and hypoglycaemia in adults [11, 12, 13]. Positron emission tomography [14] and neurophysiological event-related potential (P300) studies [15] have also failed to show more brain dysfunction in diabetic patients who have had repeated and/or severe episodes of hypoglycaemia. On the other hand, increasing evidence suggests that chronic hyperglycaemia may induce a "central neuropathy" that is characterized by mental slowing and by longer BAEP latencies, and is associated with poor metabolic control [5, 7]. Our earlier work examined this issue in a cohort of middle-aged adults who developed diabetes before 18 years of age and had this disease for more than 26 years, on average. Not only were our diabetic subjects impaired on tasks requiring psychomotor speed or spatial information processing, but individuals with one or more biomedical complication showed more cognitive impairment than those without complications. Of the biomedical variables examined, clinically significant distal symmetrical polyneuropathy (DSP) and/or elevated glycosylated haemoglobin values were most strongly associated with psychomotor slowing $[5,16]$.

If poor metabolic control leads to both biomedical and neurocognitive complications, one would predict that over time, diabetic adults would show increasingly greater cognitive impairment which would be associated with the occurrence of more, and/or more severe hyperglycaemia-associated complications. We thus conducted the following study to answer three broad questions. Do diabetic adults show a progressive decline in cognitive functioning over time? Do these changes occur in all cognitive domains, or are some cognitive abilities more likely to decline than others? If cognitive deterioration occurs, is it related to the occurrence of new diabetes-associated biomedical complications? To address those issues, we reevaluated the neuropsychological and medical status of diabetic patients and non-diabetic subjects first seen by us approximately 7 years earlier, compared their current performance to their initial assessment results, and evaluated the relationship between cognitive change and the incidence of clinically significant complications.

\section{Subjects and Methods}

Participants. Diabetic patients were originally selected randomly from the Pittsburgh Epidemiology of Diabetes Complications (EDC) study population [17]. EDC subjects were drawn from the Children's Hospital of Pittsburgh IDDM Registry which is a childhood onset $(<17$ year) cohort of Type 1 diabetic individuals diagnosed or seen within 1 year of diagno- sis at Children's Hospital of Pittsburgh between 1950 and 1980. Non-diabetic participants were recruited by asking each diabetic patient to name a spouse, sibling, significant other, or close friend. In our original study, a total of 181 diabetic adults and 146 non-diabetic adults were evaluated, all of whom were between the ages of 18 and 55 years (mean $=33.9 \pm 6.5 \mathrm{yrs}$ ). At follow-up, 34 diabetic patients could not be located or had moved out of state. An additional eight diabetic subjects had died, six were too ill to be tested, two were diagnosed with major current psychiatric disorders, and 28 declined to participate, leaving 103. Of the original 146 control subjects, 44 declined to participate and 45 could not be located or had moved out of state, leaving 57. Within each group, those who participated and those who refused or were lost to follow-up did not differ on measures of age, education, depressive symptomatology or Full Scale IQ-with one exception. Control subjects who were lost to follow-up or relocated were younger than those who participated $(32.1 \pm 7.1$ vs $34.9 \pm 7.0 ; p<0.05)$. Also noteworthy is the fact that a relatively large proportion of diabetic subjects who had DSP at baseline did not participate in the follow-up evaluation. Although $41 \%$ of the entire original diabetic cohort met criteria for DSP, nearly half were not assessed by us at follow-up, because they were deceased ( $n=7)$, too ill $(n=6)$, had moved away or were lost to follow-up $(n=10)$, or declined $(n=13)$.

This study focuses on 103 diabetic patients and 57 non-diabetic adults who were evaluated twice. None had a current or past history of chronic alcohol or drug abuse, a head injury with a loss of consciousness that exceeded $30 \mathrm{~min}$, or a current psychiatric disorder. Both the original and follow-up protocols had been reviewed and approved by the University of Pittsburgh Institutional Review Board, and written informed consent was obtained from subjects, all of whom were paid for their participation.

Medical variables. Diabetic subjects were examined biennially by EDC staff and received a detailed medical examination from a trained internist with appropriate laboratory tests to assess the presence of micro- and macrovascular complications and co-morbid disorders. This methodology is described elsewhere [18]. Non-diabetic comparison subjects completed a similar but abbreviated medical examination at the EDC Research Centre to ensure that none met criteria for diabetes [19].

Stable glycosylated haemoglobin $\left(\mathrm{HbA}_{1}\right)$ was measured with automatic high performance liquid chromatography (Diamat, BioRad, Hercules, Calif., USA); the normal range is 4.9 to $7.3 \%$. Blood pressure was measured with a random zero sphygmomanometer according to the Hypertension Detection and Follow-up Program protocol, after a 5 min rest [20]; the mean of the second and third readings was used. Vibratory thresholds were measured on the plantar surface of the great toe on the dominant side of the body, using the Vibratron II device (Physitemp Instruments, Clifton, N.J., USA). A forcedchoice procedure with up to 20 trials was used to assess large sensory nerve fibres [21]. Visual acuity was assessed at a distance of 4 metres from the eye charts, with different charts used for the two eyes, according to procedures adopted by the DCCT [22].

Retinopathy was assessed in diabetic subjects with stereoscopic color fundus photographs in three standardized fields. Photographs were graded at the Wisconsin Reading Centre using the Wisconsin Epidemiologic Study of Diabetic Retinopathy Classification and Grading System [23]. Proliferative diabetic retinopathy (PDR) was defined as grade 60 or higher in one eye or a grade less than 60 but with panretinal photocoagulation scars consistent with laser therapy. Overt Nephropathy $(\mathrm{ON})$ was defined as an albumin excretion rate greater than 
Table 1. Demographic and biomedical characteristics of both samples at follow-up assessment

\begin{tabular}{lccc}
\hline & Diabetic Subjects & Control Subjects & $p$ \\
\hline Number & 103 & 57 & 0.70 \\
\% Female & $58.3 \%$ & $1.8 \%$ & 0.66 \\
\% African American & $2.9 \%$ & $41.8 \pm 7.1$ & 0.18 \\
Age (years) & $40.4 \pm 6.2$ & $14.3 \pm 2.3$ & 0.67 \\
Years of Education & $14.5 \pm 2.4$ & $6.9 \pm 2.2$ & 0.14 \\
Years between assessments & $7.4 \pm 1.6$ & $101.7 \pm 11.0$ & 0.24 \\
WAIS-R Full Scale IQ & $99.7 \pm 10.0$ & $52.7 \pm 9.3$ & 0.34 \\
SCL-90 Depression T Score & $54.3 \pm 11.9$ & $54.4 \%$ & 0.55 \\
Any alcohol in past month (\%) & $49.5 \%$ & $3.1 \pm 2.8$ & 0.84 \\
Drinks per week (in drinkers) & $3.2 \pm 3.4$ & $110.9 \pm 14.7$ & 0.0001 \\
Haemoglobin A & $10.0 \pm 1.5$ & $71.9 \pm 10.8$ & 0.001 \\
Systolic Blood Pressure (mm Hg) & $120.1 \pm 18.7$ & $2.3 \pm 1.0$ & 0.33 \\
Diastolic Blood Pressure (mm Hg) & $70.0 \pm 11.6$ & & 0.0001 \\
Great toe vibratory thresholds & $6.1 \pm 4.5$ & & \\
Duration of diabetes (years) & $31.5 \pm 7.0$ & $8.9 \pm 3.9$ & \\
Age at Diagnosis (years) & & & \\
\hline
\end{tabular}

$200 \mu \mathrm{g} / \mathrm{min}$ in at least two of three timed urine samples, or end-stage renal disease (renal dialysis or transplant). Distal symmetric polyneuropathy (DSP) was based on a clinical neurological evaluation carried out by a trained internist and defined according to the DCCT criteria [24] as the presence of two or more of the following: symptoms consistent with DSP, decreased or absent tendon reflexes, or signs of sensory loss, all in the absence of other known etiologies. Autonomic neuropathy (AN) was determined from the mean value of the longest RR interval during expiration and the shortest RR interval during inspiration based on a continuous ECG recording during a $1 \mathrm{~min}$ breathing procedure. $\mathrm{AN}$ was considered to be present if the E/I ratio was less than or equal to 1.1 [25]. Peripheral vascular disease (PVD) was defined as an ankleto-arm blood pressure ratio less than 0.9 in any one of the four distal leg arteries (tibial anterior or dorsalis pedis), or by amputation for vascular insufficiency. Coronary artery disease (CAD) was defined as angina diagnosed by a clinic physician; myocardial infarction confirmed by Q-waves on electrocardiogram and/or validated hospital records, or coronary artery stenosis greater than or equal to $50 \%$ confirmed by angiography.

Neuropsychological assessment. Detailed descriptions of each measure can be found elsewhere [26, 27]. Learning and memory skills were assessed with the Verbal Paired-Associate Learning Test, the Symbol-Digit Paired-Associate Learning Test, the Four-Word Short-Term Memory Test, and the Rey Complex Figure Memory Test. Problem-solving and spatial organization was assessed with the Tactual Performance Test, the Object Assembly and Block Design subtests from the Wechsler Adult Intelligence Scale, Revised (WAIS-R), and the Wisconsin Card Sorting Test. Psychomotor efficiency was measured with the completion time score from the Digit Vigilance Test, the WAIS-R Digit Symbol Substitution Test, the Grooved Pegboard, and Part B of the Trail Making Test. Intelligence was assessed at baseline with the WAIS-R. Symptoms of depression over the previous month were ascertained from the Symptom-Checklist 90-R (SCL-90) [28]. Acute emotional state at the time of testing was evaluated with the Profile of Mood States [29]. To ensure that neuropsychological test results were not influenced by ambient hypoglycaemia, a fingerstick blood glucose reading was obtained before cognitive assessment; subjects with values less than $3.9 \mathrm{mmol} / \mathrm{l}$ were treated with a snack and re-tested.
Statistical analyses. Descriptive univariate between-group comparisons were assessed with Student's $t$ test, analysis of variance (ANOVA) with planned comparisons, chi square, or Wilcoxon, as appropriate. A $p$ value less than or equal to 0.05 was considered to be statistically significant. Factor analysis techniques (principal components analysis with varimax rotation) were used to cluster the large number of neuropsychological variables into a manageable number of broad cognitive domains. For each test, raw scores were converted into standardized $\mathrm{z}$ scores $(\mathrm{M}=0 ; \mathrm{SD}=1)$, using the mean and standard deviation values from the non-diabetic comparison group at time 1 . Domain $\mathrm{z}$ scores were calculated as the mean $\mathrm{z}$ value of the cognitive tests assigned to that specific domain. A two-factor (Group $\times$ Time) repeated measures ANOVA procedure was used to evaluate change in functioning over time within each domain. If statistically significant GroupxTime interactions were found, logistic regression techniques were used with diabetic subjects to identify salient predictors of significant cognitive deterioration. All statistical analyses were carried out using SPSS software (version 11 for Windows; SPSS, Chicago, Ill., USA).

\section{Results}

Demographic and biomedical characteristics of the two groups are summarized in Table 1. Both groups were of average intelligence, moderately well-educated, and without symptoms of depression. As expected, the diabetic group showed elevated glycosylated haemoglobin values, systolic blood pressures, and great toe vibratory thresholds.

Biomedical complications. At time 1, this group of diabetic patients had high rates of microvascular complications (AN: $23 \%$ of sample; DSP: 27\%; ON: $29 \%$; PDR: 44\%), but relatively low rates of macrovascular complications (CAD: 9\%; PVD: 5\%). Over the ensuing 7 year follow-up period, incidence of new microvascular complications increased appreciably (AN: 30\%; DSP: 22\%; ON: 9\%; PDR: 16\%). The incidence 
of microvascular complications was intercorrelated: incident PDR correlated with the incidence of DSP $(\mathrm{r}=.35)$ and $\mathrm{ON}(\mathrm{r}=.25)$; incident AN correlated with incident DSP $(\mathrm{r}=.21)$. Occurrence of new macrovascular complications was also high (CAD: 21\%; PVD: $15 \%)$. Prevalence data at follow-up indicated high rates of comorbid conditions: $51.5 \%$ of the entire sample had three or more micro- and/or macrovascular complications, whereas only $19 \%$ of the sample were without any complication.

Cognitive performance and complications. Principal components factor analysis assigned the test scores to three cognitive domains: Learning and memory, spatial and problem-solving skills, and psychomotor efficiency. Together, these accounted for $63 \%$ of the total variance. Results from the ANOVAs showed that statistically significant deterioration was evident only on the psychomotor efficiency measures. There was a main effect of Group ( $\mathrm{F}=12.77 ; p=0.0001)$, as well as a main effect of Time $(\mathrm{F}=5.66 ; p=0.019)$ and a Group $\mathrm{x}$ Time interaction $(\mathrm{F}=8.01 ; p=0.005)$. That is, diabetic subjects were consistently slower than control subjects at both assessments, and showed a greater degree of decline in functioning from time 1 to time 2 , as compared to control subjects (Fig. 1). In contrast, both groups performed nearly identically on measures of learning and memory (all $\mathrm{F} s<1$; p $s>0.75$ ) and on measures of spatial and problem-solving skills (all Fs $\leq 1$ ).

Hierarchical logistic regression techniques were used with the diabetic sample group to evaluate the predictors of psychomotor deterioration (dichotomized as a decline in summary psychomotor $\mathrm{z}$ score $\geq 0.5 \mathrm{SD}$ unit). Using that operational definition, $39 \%$ of the diabetic group sample and $9 \%$ of the non-diabetic group sample met criteria for psychomotor decline $(p<0.0001)$. Psychomotor change was not related to sensory abilities, medication use, or emotional state during testing. Compared to those who did not change, diabetic patients whose psychomotor efficiency declined by at least 0.5 standard deviation units were similar on measures of acute tension $(\mathrm{t}=0.69 ; p>0.40)$ and acute depression ( $\mathrm{t}=1.10 ; p>0.35)$, visual acuity $\left(\chi^{2}=0.5 ; p>0.40\right)$, and use of hypertensive medication $\left(\chi^{2}=2.57 ; p>0.10\right)$.

The use of a hierarchical regression procedure allows us to systematically examine the independent contribution of each block of variables, after taking into account the effects of the previous block of variables. This analysis examines the effects of three types of predictor variables on change in psychomotor speed over a 7 year period: time 1 variables (e.g., baseline psychomotor score; prevalence of micro- and macrovascular complications), time 2 variables (e.g., incidence of new micro- and macrovascular complications), and other diabetes-associated variables (e.g., elevated blood pressure, duration of disease, recent metabolic control). Predictor variables were entered in

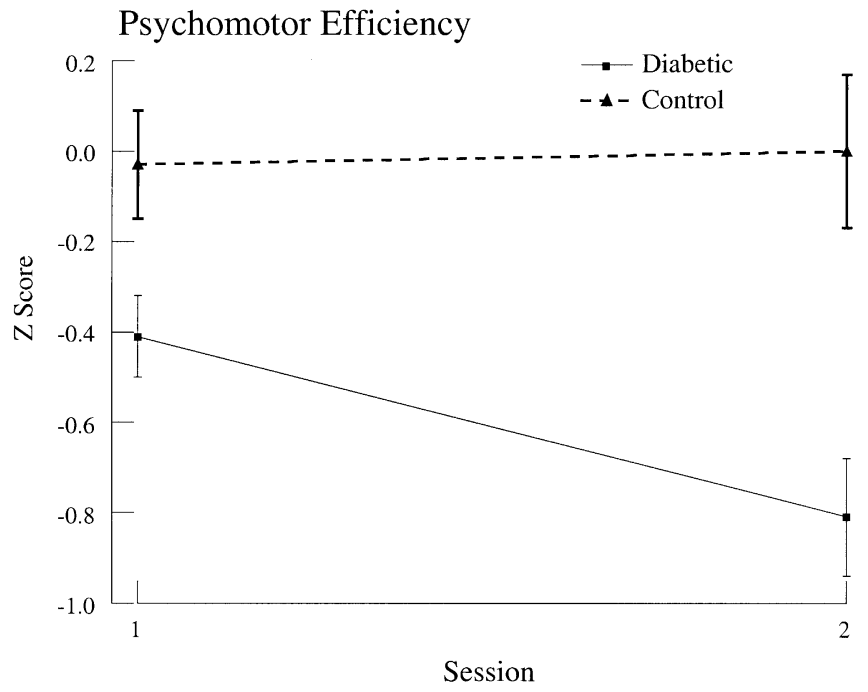

Fig. 1. Changes in psychomotor efficiency (expressed in $\mathrm{Z}$-score units; $\mathrm{M} \pm \mathrm{SEM}$ ) for participants with (solid line) and without (dashed line) diabetes who were evaluated at two points in time, approximately 7 years apart

eight separate blocks: (i) time 1 psychomotor summary score; (ii) time 1 prevalence of microvascular complications (AN, DSP, nephropathy, retinopathy); (iii) time 1 prevalence of macrovascular complications (CAD, PVD); (iv) time 2 incidence of microvascular complications; (v) time 2 incidence of macrovascular complications; (vi) time 2 systolic blood pressure; (vii) duration of diabetes; (viii) time 2 metabolic control $\left(\mathrm{HbA}_{1}\right)$. Systolic blood pressure was included in these analyses because prior studies have shown that elevated blood pressure can affect neuropsychological test performance in adults with diabetes.

Our analyses showed that pre-existing microvascular complications at time 1 predicted slowing (Block 2: $\chi^{2}=9.2 ; p=0.057$; Nagelkerke $\mathrm{R}^{2}$ change $=0.12$ ), but pre-existing macrovascular complications did not (Block 3: $\chi^{2}<1$ ). Both incident microvascular complications at time 2 (Block 4: $\chi^{2}=14.2 ; p=0.007 ; \mathrm{R}^{2}$ change $=0.17)$ and incident macrovascular complications (Block 5: $\chi^{2}=6.4 ; p=0.04 ; \mathrm{R}^{2}$ change $=0.07$ ) were predictors of psychomotor slowing at time 2 , as were systolic blood pressure at time 2 (Block 7: $\chi^{2}=9.7$; $p=0.002 ; \mathrm{R}^{2}$ change $=0.09$ ) and duration of diabetes (Block 7: $\chi^{2}=9.1 ; p=0.003 ; \mathrm{R}^{2}$ change $=0.08$ ). The addition of $\mathrm{HbA}_{1}$ at time 2 did not contribute $\left(\chi^{2}<1\right)$ and was deleted from the final model. Coefficients for the final logistic regression model are summarized in Table 2. This model, which included seven blocks of variables, correctly classified $83 \%$ of subjects and explained $53 \%$ of the variance. When the analysis was repeated, but with the substitution of total number of micro- and macrovascular complications for the presence or absence of specific complications, a similar pattern of results was obtained (full model not shown). Both the number of time 1 prevalent $\left(\chi^{2}=8.2 ; p=0.004\right.$; 
Table 2. Summary of coefficients from hierarchical logistic regression analysis

\begin{tabular}{lrllll}
\hline & B & S.E. & Wald & Odds Ratio & C.I. \\
\hline Time 1 Psychomotor Speed & -0.716 & 0.41 & 3.11 & 0.49 & $0.23,1.09$ \\
Time 1 AN & 1.139 & 0.86 & 1.75 & 3.12 & $0.58,16.84$ \\
Time 1 DSP & 0.109 & 1.04 & 0.01 & 1.12 & $0.14,8.63$ \\
Time 1 ON & 0.377 & 0.83 & 0.21 & 1.46 & $0.29,7.37$ \\
Time 1 PDR & 0.437 & 0.75 & 0.39 & 1.61 & $0.37,6.98$ \\
Time 1 CAD & -0.904 & 1.26 & 0.51 & 0.41 & $0.03,4.82$ \\
Time 1 PVD & -1.634 & 1.40 & 1.35 & 0.19 & $0.01,3.06$ \\
Time 2 Incident AN & $\mathbf{1 . 7 3 4}$ & $\mathbf{0 . 7 6}$ & $\mathbf{5 . 2 3}$ & $\mathbf{5 . 6 6}$ & 0.917 \\
Time 2 Incident DSP & -1.541 & 0.89 & 2.96 & 0.21 & 0.528 \\
Time 2 Incident ON & 0.135 & 1.28 & 0.01 & 1.14 & $0.04,1.24$ \\
Time 2 Incident PDR & $\mathbf{2 . 8 7 7}$ & $\mathbf{0 . 9 9}$ & $\mathbf{8 . 3 5}$ & $\mathbf{1 7 . 7 7}$ & $0.09,14.10$ \\
Time 2 Incident CAD & -0.209 & 0.80 & 0.68 & 0.81 & $\mathbf{2 . 5 3 , 1 2 5 . 0 3}$ \\
Time 2 Incident PVD & 1.027 & 0.87 & 1.38 & 2.79 & $0.17,3.89$ \\
Follow-up SBP & $\mathbf{0 . 0 4 6}$ & $\mathbf{0 . 0 2}$ & $\mathbf{5 . 2 9}$ & $\mathbf{1 . 0 5}$ & $0.50,15.52$ \\
Duration of Diabetes & $\mathbf{0 . 1 5 1}$ & $\mathbf{0 . 0 6}$ & $\mathbf{7 . 2 4}$ & $\mathbf{1 . 1 6}$ & $\mathbf{0 . 0 2 2}$ \\
\hline
\end{tabular}

Table 3. Relationship between change in biomedical complications over time (no complication at either assessment; new complication at time 2; complication present at time 1) and psychomotor efficiency (mean; confidence interval) expressed as a standardized ( $\mathrm{z}$ ) change score between first and second assessment

\begin{tabular}{lllll}
\hline & AN & DSP & ON & PDR \\
\hline No complication at either time & $-.22(.09, .34)$ & $-.31(.18, .45)$ & $-.31(.19, .43)$ & $-.22(.09, .36)$ \\
Incident complication: Time 2 & $-.57(.38, .75)$ & $-.39(.18, .61)$ & $-.44(.04, .85)$ & $-.56(.29, .84)$ \\
Complication present: Time 1 & $-.56(.30, .81)$ & $-.57(.34, .80)$ & $-.58(.33, .82)$ & $-.50(.33, .66)$ \\
\hline
\end{tabular}

$\mathrm{R}^{2}$ change $\left.=0.11\right)$ and time 2 incident $\left(\chi^{2}=6.4 ; p=0.01 ;\right.$ $\mathrm{R}^{2}$ change $\left.=0.08\right)$ microvascular complications were associated with psychomotor slowing, as was the number of incident macrovascular complications $\left(\chi^{2}=4.3\right.$; $p=0.04 ; \mathrm{R}^{2}$ change $=0.05$ ).

To assess the magnitude of psychomotor slowing associated with the occurrence of biomedical complications over time, a subsequent analysis compared individuals with no complication at both time points, to those with at least one complication already present by the time 1 assessment, and to those who first were diagnosed with the complication between time 1 and time 2. Individuals who developed retinopathy between time 1 and time 2 showed greater psychomotor deterioration than those with no retinopathy at either assessment $(p<0.02)$, but earned scores that were comparable to individuals with pre-existing retinopathy at time 1 (Table 3). These data show that a diagnosis of PDR is associated with about a third of a standard deviation unit decline in psychomotor efficiency. A similar pattern of results is seen when one examines the relationship between onset of autonomic neuropathy and changes in psychomotor function. In contrast, the incidence of either DSP or ON by time 2 does not predict slowing at time $2(p>0.05)$, but the presence of either of those complications at time 1 does $(p<0.05)$.

Given the well-established relationship between glycosylated haemoglobin values and biomedical complications, the failure of our multivariate analyses to show any association between current $\mathrm{HbA}_{1}$ and cognitive change was unexpected. To evaluate the possibility that subjects with a recent onset of complications had altered their diabetes management or otherwise improved their metabolic control, we conducted a series of exploratory univariate analyses that compared $\mathrm{HbA}_{1}$ values at baseline and follow-up for subjects with and without new complications. The strong relationship between retinopathy and cognitive dysfunction in this study led us to focus on the development of PDR. We compared the three subgroups, as described above: (i) no PDR at either assessment; (ii) PDR present at both assessments; (iii) incident PDRdeveloping after the first assessment. Glycosylated haemoglobin values at baseline (assessment 1) were higher for subjects who subsequently developed PDR by assessment $2(11.6 \pm 1.9 \%)$, as compared to those with no PDR at either assessment $(9.9 \pm 1.4 ; \mathrm{t}=2.9$; $p<0.007)$ and those with PDR at the time of study entry $(10.7 \pm 1.8 ; \mathrm{t}=1.77 ; p=0.08)$. Measures of $\mathrm{HbA}_{1}$ obtained at follow-up indicated that individuals with an incidence of PDR between the first and second assessment showed a decline in $\mathrm{HbA}_{1}$ values (from $11.6 \pm 1.9 \%$ to $10.1 \pm 1.3 \% ; \mathrm{t}=2.42 ; p=0.03$ ); the other two groups showed no change over time. 


\section{Discussion}

Our evaluation of adults with Type 1 diabetes, followed over a 7-year period, leads us to conclude that the development of clinically significant biomedical complications correlates with, and could contribute to, the psychomotor slowing which is characteristically seen in this patient population. We and others have previously shown that mental and motor slowing is a nearly ubiquitous sequela of both Type 1 and Type 2 diabetes $[2,3,5,30,31]$. This prospective assessment confirms those earlier cross-sectional findings by showing that over time, $39 \%$ of our diabetic sample showed a marked (more than 0.5 SD units) decline on a composite measure of psychomotor efficiency, whereas only $9 \%$ of our non-diabetic comparison group showed a similar decline. It is noteworthy that cognitive change in this patient cohort was limited to the psychomotor domain. Neither learning and memory skills, nor visuospatial and problem-solving skills, were found to deteriorate.

Our data also provide evidence that the occurrence of new diabetes-associated biomedical complications was associated with deterioration in psychomotor efficiency. Within our sample, the incidence of two microvascular complications had the greatest effect on psychomotor decline: proliferative diabetic retinopathy, and autonomic neuropathy. The magnitude of this effect was large, with each incident complication being associated with a decline of more than a half standard deviation, on average. Additional salient predictors of decline included longer duration of diabetes, and elevated blood pressure. Together, these variables accounted for $40 \%$ of the variance. In contrast, glycosylated haemoglobin values measured at time 2 did not contribute further to the explanatory value of this model. Our failure to find an isomorphic relationship between $\mathrm{HbA}_{1}$ levels and cognitive decline may reflect the possibility - as supported in our exploratory analyses - that the development of a clinically significant complication like retinopathy could be accompanied by improvements in metabolic control that might be secondary to changes in diabetes management.

A substantial body of literature has suggested that chronic hyperglycaemia and/or the occurrence of complications could directly or indirectly affect brain function [32, 33]. As virtually all of this work has been conducted in adults with Type 2 diabetes, it is unlikely that those cognitive changes can be attributed to recurrent hypoglycaemia, given the very low rates of hypoglycaemia in patients with Type 2 diabetes [34]. In longitudinal studies, performance decrements over a 3- to 7-year follow-up period have been found most consistently on psychomotor tasks like the Digit Symbol Substitution Test (DSST), and these effects persist, even after controlling statistically for other cardiovascular risk factors $[35,36,37,38]$.
Aside from our own work, only two studies have followed Type 1 diabetic patients over time. Unfortunately, because the focus of both the DCCT and the Stockholm Diabetes Intervention Study was on the cognitive effects of hypoglycaemia, analyses did not directly examine the possibility that duration of diabetes, presence of complications, or other markers of poor metabolic control might adversely affect cognition. As neither included a group of demographicallysimilar non-diabetic subjects, analyses could not take into account the potentially confounding effects of normal age-related changes in cognitive performance or of practice. Nevertheless, comparisons between intensively and conventionally treated participants in the DCCT indicated that over a 6-year follow-up period, those treated conventionally with one or two daily injections showed statistically significant (albeit slight) worsening over time on measures of motor speed, and this was directly associated with higher glycosylated haemoglobin values-and by implication, with more, or more severe, microvascular complications [12].

Previous cross-sectional research has identified two microvascular complications-retinopathy and peripheral neuropathy-as predictors of cognitive dysfunction in adults with diabetes [5, 39]. For example, a recent study of young adults with Type 1 diabetes of relatively short duration showed that background diabetic retinopathy was associated with poorer performance on measures of fluid intelligence, information processing efficiency, and attention, and was also associated with higher rates of brain structure abnormalities (white matter hyperintensities) on MRI scans [39]. Others have reported the existence of a similar relationship between retinopathy and cognitive dysfunction, but in the absence of diabetes. A study of more than 8500 middle-aged adults without stroke, found retinal microaneurysms and/or haemorrhages to be associated with lower scores on measures of mental efficiency (e.g., DSST) and delayed memory, even after controlling for education, diabetes, blood pressure, carotid intima-media thickness and other potential risk factors [40]. Arguing that examination of the retinal arterioles provides a non-invasive assessment of the cerebral microcirculation, those authors concluded that retinopathy-associated cognitive impairment could have a vascular component, and speculated that this could reflect a disruption of the bloodbrain barrier [40] and/or the development of white matter lesions [41].

Distal symmetric polyneuropathy has also been found to predict the degree of cognitive slowing in adults with Type 1 diabetes [5], and like both retinopathy and nephropathy, this neurological complication is now thought to be largely vascular in origin [42]. If the effects of these microvascular complications on the central nervous system (CNS) are additive or synergistic, one might expect to see a relationship between the number of complications and the magnitude 
of cognitive change. Results from our study are consistent with that possibility: the greater the number of incident complications, the greater the magnitude of psychomotor slowing. Although it is tempting to attribute diabetes-associated cognitive dysfunction largely to the accretion of microvascular changes in the CNS, other plausible underlying mechanisms include alterations in aminergic neurotransmitter pathways [43], and/or the accumulation of advanced glycosylation end products in the CNS [44].

Elevated blood pressure is a common comorbid condition in patients with diabetes, which was also found by us to be an independent predictor of cognitive decline. Multiple studies have shown that elevated blood pressure not only predicts later-life changes on measures of brain morphology $[45,46]$ and cognitive function $[45,47]$ - particularly on speeded tests like the DSST [37, 48], but could act synergistically with diabetes [49] or insulin resistance [50] to increase the severity of neurocognitive impairment. Higher blood pressure might adversely affect mental efficiency via vascular and/or neural pathways. For example, during the performance of increasingly difficult cognitive tasks, hypertensive adults show a relatively smaller increase in $\mathrm{rCBF}$, as compared to normotensives [51]. Since cognitive effort normally triggers an increase in cerebral blood supply in response to increasing metabolic demands, any reduction in vascular reactivity is likely to induce a decline in mental efficiency. The greatly increased incidence of white matter lesions in adults with hypertension [52, 53] could also underlie the decline in mental efficiency, since the presence of white matter lesions is associated with slower performance on psychomotor tasks like the DSST $[54,55]$. Whether those declines reflect neural changes secondary to demyelination [56] or to the haemodynamic abnormalities associated with white matter lesions [57] remains to be determined.

In our earlier cross-sectional work, we speculated that diabetes-associated mental slowing is a consequence of a "central neuropathy" which is characterized by diffusely-distributed cerebral white matter dysfunction and is associated with hyperglycaemiainduced complications [5]. Results from the current study are consistent with that hypothesis. We found that the incidence of complications-particularly retinopathy, predicted poorer performance on measures of psychomotor efficiency that are known to be adversely affected by cerebral white matter diseases like multiple sclerosis, Binswanger's disease, and alcoholism [58]. Although we did not use either neurophysiological or neuroimaging techniques to assess CNS integrity, others have reported relationships between diabetes-related biomedical complications and both slower BAEP conduction velocities $[59,60]$ and increased incidence of white matter hyperintensities on MRI [39]. A more definitive test of the white matter hypothesis will require the pairing of traditional neuropsychologi- cal and electrophysiological measures of CNS function with newer imaging modalities, like diffusion tensor imaging [61], to map the integrity of white matter tracts in diabetic adults.

In conclusion, our data shows that in young and middle-aged adults with Type 1 diabetes, cognitive function declines over time and these changes are linked to the development of microvascular complications and to elevations in blood pressure. If, as our findings suggest, microangiopathy is a leading cause, or marker, of neurocognitive decline, then any therapeutic intervention that reduces the risk of complications may have a similarly salutary effect on the CNS, and induce a corresponding reduction in the risk of developing neurocognitive complications in diabetic patients.

Acknowledgements. This research was supported in part by National Institute of Health Grant 5 RO1 DK39629.

\section{References}

1. Biessels G-J (1999) Cerebral complications of diabetes: Clinical findings and pathogenetic mechanisms. Neth $\mathrm{J}$ Med 54:35-45

2. Deary IJ, Langan SJ, Graham KS, Hepburn D, Frier BM (1992) Recurrent severe hypoglycemia, intelligence, and speed of information processing. Intelligence 16:337-359

3. Sachon C, Grimaldi A, Digy JP, Pillon B, Dubois B, Thervet F (1992) Cognitive function, insulin-dependent diabetes and hypoglycaemia. J Intern Med 231:471-475

4. Wredling R, Levander S, Adamson U, Lins PE (1990) Permanent neuropsychological impairment after recurrent episodes of severe hypoglycaemia in man. Diabetologia 33:152-157

5. Ryan CM, Williams TM, Orchard TJ, Finegold DN (1992) Psychomotor slowing is associated with distal symmetrical polyneuropathy in adults with diabetes mellitus. Diabetes 41:107-113

6. Perros P, Deary IJ, Sellar RJ, Best JJK, Frier BM (1997) Brain abnormalities demonstrated by magnetic resonance imaging in adult IDDM patients with and without a history of recurrent severe hypoglycemia. Diabetes Care 20:10131018

7. Dejgaard A, Gade A, Larsson H, Balle V, Parving A, Parving H (1991) Evidence for diabetic encephalopathy. Diabet Med 8:162-167

8. Quirce R, Carril JM, Jiménez-Bonilla JF, Amado JA, Gutiérrez-Mendiguchía C, Banzo I, Blanco I, Uriarte I, Montero A (1997) Semi-quantitative assessment of cerebral blood flow with 99mTc-HMPAO SPET in type 1 diabetic patients with no clinical history of cerebrovascular disease. Eur J Nucl Med 24:1507-1513

9. Perros P, Deary IJ (1999) Long-term effects of hypoglycaemia on cognitive function and the brain in diabetes. In: Frier BM, Fisher BM (eds) Hypoglycaemia in clinical diabetes. Wiley, Chichester, pp 187-210

10. Deary I, Crawford J, Hepburn DA, Langan SJ, Blackmore LM, Frier BM (1993) Severe hypoglycemia and intelligence in adult patients with insulin-treated diabetes. Diabetes $42: 341-344$

11. Austin EJ, Deary IJ (1999) Effects of repeated hypoglycemia on cognitive function. Diabetes Care 22:1273-1277 
12. Diabetes Control and Complications Trial Research Group (1996) Effects of intensive diabetes therapy on neuropsychological function in adults in the Diabetes Control and Complications Trial. Ann Intern Med 124:379-388

13. Reichard P, Berglund B, Britz A, Levander S, Rosenqvist U (1991) Hypoglycemic episodes during intensified insulin treatment: increased frequency but no effect on cognitive function. J Intern Med 229:9-16

14. Chabriat H, Sachon C, Levasseur M, Grimaldi A, Pappata S, Rougemont D, Masure MC, De Rocondo A, Samson Y (1994) Brain metabolism after recurrent insulin-induced hypoglycemic episodes: A PET study. J Neurol Neurosurg Psychiatry 57:1360-1365

15. Kramer L, Fasching P, Madl C, Schneider B, Damjancic P, Waldhäusl W, Irsigler K, Grimm G (1998) Previous episodes of hypoglycemic coma are not associated with permanent cognitive brain dysfunction in IDDM patients on intensive insulin treatment. Diabetes 47:1909-1914

16. Ryan CM, Williams TM, Finegold DN, Orchard TJ (1993) Cognitive dysfunction in adults with Type 1 (insulindependent) diabetes mellitus of long duration: effects of recurrent hypoglycaemia and other chronic complications. Diabetologia 36:329-334

17. Orchard TJ, Dorman JS, Maser RE, Becker DJ, Drash AL, Ellis D, Laporte RE, Kuller LH (1990) Prevalence of complications in IDDM by sex and duration: Pittsburgh Epidemiology of Complications Study II. Diabetes 39:1116-1124

18. Orchard TJ, Forrest KY-Z, Kuller LH, Becker DJ (2001) Lipid and blood pressure treatment goals for Type 1 diabetes: 10-year incidence data from the Pittsburgh Epidemiology of Diabetes Complications Study. Diabetes Care 24:1053-1059

19. American Diabetes Association (1999) Screening for Type 2 diabetes. Diabetes Care 22:S20-S23

20. Borhani NO, Kass EH, Langford HG, Payne GH, Remington RD, Stamler J (1976) The hypertension detection and follow-up program. Prev Med 5:207-215

21. Maser RE, Nielsen VK, Bass EB, Manjoo Q, Dorman JS, Kelsey SF, Becker DJ, Orchard TJ (1989) Measuring diabetic neuropathy: Assessment and comparison of clinical examination and quantitative sensory testing. Diabetes Care 12:270-275

22. Diabetes Control and Complications Trial Research Group (1986) The Diabetes Control and Complications Trial (DCCT): design and methodologic considerations for the feasibility phase. Diabetes 35:530-545

23. Early Treatment of Diabetic Retinopathy Study Coordinating Center (1980) Manual of operations. University of Maryland School of Medicine, Baltimore, MD

24. Diabetes Control and Complications Trial Research Group (1988) Factors in development of diabetic neuropathy: Baseline analysis of neuropathy in feasibility phase of Diabetes Control and Complications Trial (DCCT). Diabetes 37:476-481

25. Stella P, Ellis D, Maser RE, Orchard TJ (2000) Cardiac autonomic neuropathy (expiration and inspiration ratio) in type 1 diabetes: Incidence and predictors. J Diabetes Complications 14:1-6

26. Ryan CM (1994) Measures of cognitive function. In: Bradley C (ed.) Handbook of psychology and diabetes. Harwood, Chur, Switzerland, pp 191-219

27. Mitrushina MN, Boone KB, D'Elia LF (1999) Handbook of normative data for neuropsychological assessment. Oxford University Press, New York

28. Derogatis LR (1983) SCL-90-R manual: administration, scoring \& procedures. Clinical Psychometric Research, Towson, MD
29. McNair DM, Lorr M, Droppleman LF (1981) EDITS manual for the profile of mood states. Educational and Industrial Service, San Diego, CA

30. Ryan C, Vega A, Longstreet C, Drash L (1984) Neuropsychological changes in adolescents with insulin-dependent diabetes mellitus. J Consult Clin Psychol 52:335342

31. Ryan CM, Geckle MO (2000) Circumscribed cognitive dysfunction in middle-aged adults with type 2 diabetes. Diabetes Care 23:1486-1493

32. Stewart RJ, Liolitsa D (1999) Type 2 diabetes mellitus, cognitive impairment and dementia. Diabet Med 16:93112

33. Strachan MWJ, Deary IJ, Ewing FME, Frier BM (1997) Is Type 2 (non-insulin dependent) diabetes mellitus associated with an increased risk of cognitive dysfunction? Diabetes Care 20:438-445

34. Miller CD, Phillips LS, Ziemer DC, Gallina DL, Cook CB, El-Kebbi IM (2001) Hypoglycemia in patients with Type 2 diabetes mellitus. Arch Intern Med 161:1653-1659

35. Fontbonne A, Ducimetiere P, Berr C, Alperovitch A (2001) Changes in cognitive abilities over a 4-year period are unfavorably affected in elderly diabetic subjects: results of the Epidemiology of Vascular Aging Study. Diabetes Care 24:366-370

36. Gregg EW, Yaffe K, Cauley JA, Rolka DB, Blackwell TL, Narayan KM, Cummings SR (2000) Is diabetes associated with cognitive impairment and cognitive decline among older women? Arch Intern Med 160:174-180

37. Knopman DS, Boland LL, Mosley T, Howard G, Liao D, Szklo M, McGovern PG, Folsom AR, ARIC Study Investigators (2001) Cardiovascular risk factors and cognitive decline in middle-aged adults. Neurology 56:42-48

38. Haan MN, Shemanski L, Jagust WJ, Manolio TA, Kuller L (1999) The role of APOE $\varepsilon 4$ in modulating effects of other risk factors for cognitive decline in elderly persons. JAMA 282:40-46

39. Ferguson SC, Blane A, Perros P, McCrimmon RJ, Best JJK, Wardlaw JM, Deary IJ, Frier BM (2003) Cognitive ability and brain structure in type 1 diabetes: Relation to microangiopathy and preceding severe hypoglycemia. Diabetes 52:149-156

40. Wong TY, Klein R, Sharrett AR, Nieto FJ, Boland LL, Couper DJ, Mosley TH, Klein BEK, Hubbard LD, Szklo M (2002) Retinal microvascular abnormalities and cognitive impairment in middle-aged persons: the Atherosclerosis Risk in Communities Study. Stroke 33:1487-1492

41. Wong TY, Klein R, Sharrett AR, Couper DJ, Klein BEK, Liao D-P, Hubbard LD, Mosley TH, ARIC Investigators (2002) Cerebral white matter lesions, retinopathy, and incident clinical stroke. JAMA 288:67-74

42. Cameron NE, Eaton SEM, Cotter MA, Tesfaye S (2001) Vascular factors and metabolic interactions in the pathogenesis of diabetic neuropathy. Diabetologia 44:19731988

43. Price JC, Kelley DE, Ryan CM, Meltzer CC, Drevets WC, Mathis CA, Mazumdar S, Reynolds CF (2002) Evidence of increased serotonin-1A receptor binding in type 2 diabetes: a positron emission tomography study. Brain Res 927:97103

44. Friedman EA (1999) Advanced glycosylated end products and hyperglycemia in the pathogenesis of diabetic complications. Diabetes Care 22:B65-B71

45. Swan GE, DeCarli C, Miller BL, Reed T, Wolf PA, Jack LM, Carmelli D (1998) Association of midlife blood pressure to late-life cognitive decline and brain morphology. Neurology 51:986-993 
46. Strassburger TL, Lee H-C, Daly EM, Szczepanik J, Krausuki JS, Mentis MJ, Salerno JA, DeCarli C, Schapiro MB, Alexander GE (1997) Interactive effects of age and hypertension on volumes of brain structures. Stroke 28:1410-1417

47. Skoog I, Lernfelt B, Landahl S, Palmertz B, Andreasson L-A, Nilsson L, Persson G, Odén A, Svanborg A (1996) 15 -year longitudinal study of blood pressure and dementia. Lancet 347:1141-1145

48. Elias MF, Robbins MA, Elias PK, Streeten DHP (1998) A longitudinal study of blood pressure in relation to performance on the Wechsler Adult Intelligence Scale. Health Psychol 17:486-493

49. Elias PK, Elias MF, D’Agostino RB, Cupples LA, Wilson PW, Silbershatz H, Wolf PA (1997) NIDDM and blood pressure as risk factors for poor cognitive performance. Diabetes Care 20:1388-1395

50. Kilander L, Nyman H, Boberg M, Hansson L, Lithell H (1998) Hypertension is related to cognitive impairment: a 20-year follow-up of 999 men. Hypertension 31:780786

51. Jennings JR, Muldoon MF, Ryan CM, Mintun MA, Meltzer CC, Townsend DW, Sutton-Tyrrell K, Shapiro AP, Manuck SB (1998) Cerebral blood flow in hypertensive patients: an initial report of reduced and compensatory blood flow responses during performance of two cognitive tasks. Hypertension 31:1216-1222

52. Longstreth WT, Manolio TAAA, Burke GL, Bryan N, Jungreis CA, Enright PL, O'Leary D, Fried L (1996) Clinical correlates of white matter findings on cranial magnetic resonance imaging of 3301 elderly people: the Cardiovascular Health Study. Stroke 27:1274-1282
53. Sierra C, Sierra A de la, Mercader JM, Gómez-Angelats E, Urbano-Márquez A, Coca A (2002) Silent cerebral white matter lesions in middle-aged essential hypertensive patients. J Hypertens 20:519-524

54. Leaper SA, Murray AD, Lemmon HA, Staff RT, Deary IJ, Crawford JR, Whalley LJ (2001) Neuropsychologic correlates of brain white matter lesions depicted on MR images: 1921 Aberdeen birth cohort. Radiology 221:51-55

55. Groot JC de, Leeuw F-E de, Oudkerk M, Gijn J van, Hofman A, Jolles J, Breteler MMB (2000) Cerebral white matter lesions and cognitive function: the Rotterdam Scan Study. Ann Neurol 47:145-151

56. Miller EM (1994) Intelligence and brain myelination: a hypothesis. Pers Individ Diff 17:803-832

57. Marstrand JR, Garde E, Rostrup E, Ring P, Rosenbaum S, Mortensen EL, Larsson HBW (2002) Cerebral perfusion and cerebrovascular reactivity are reduced in white matter hyperintensities. Stroke 33:972-976

58. Filley CM (2001) The behavioral neurology of white matter. Oxford University Press, Oxford, UK

59. Virtaniemi J, Laakso M, Kärjä J, Nuutinen J, Karjalainen S (1993) Auditory brainstem latencies in type 1 (insulindependent) diabetic patients. Am J Otolaryngol 14:413418

60. Kurita A, Katayama K, Mochio S (1996) Neurophysiological evidence for altered higher brain functions in NIDDM. Diabetes Care 19:361-364

61. Pfefferbaum A, Sullivan EV, Hedehus M, Lim KO, Adalsteinsson E, Moseley M (2000) Age-related decline in brain white matter anisotropy measured with spatially corrected echo-planar diffusion tensor imaging. Magn Reson Med 44:259-266 